Revista de Investigación Lingüistica, $n .^{\circ} 21$ (2018)

ISSN: 1139-1146

Universidad de Murcia

\title{
DE LA ETNOLEXICOGRAFÍA A LA LEXICOGRAFÍA CRÍTICA
}

\author{
SusANA RODRÍGUEZ BARCIA \\ UNIVERSIDADE DE VIGO
}

Resumen: En este trabajo se presenta y establecen los límites de la lexicografía crítica como reciente disciplina dentro del ámbito de los estudios metalexicográficos. En concreto, se enmarca en el contexto general de la etnolexicografía (Fillmore 1994; Pérez 1997, 2000, 2005; Calero Vaquera 2010) con el fin de determinar su espacio propio dentro de las investigaciones lingüísticas y de destacar su relevancia en los estudios del discurso realizados con perspectiva crítica. Asimismo, también se recogen sus principios teóricos, líneas fundamentales de trabajo y claves analíticas.

Palabras clave: Lexicografía, metalexicografía, etnolexicografía, análisis crítico del discurso, ideología, discurso lexicográfico, diccionario.
From Ethnolexicography to Critical Lexicography

Abstract: In this paper the limits of critical lexicography are presented and defined as a recent discipline that is part of the field of meta-lexicographical studies. This paper falls squarely in the general framework of ethno-lexicography (Fillmore 1994; Pérez 1997, 2000, 2005; Calero Vaquera 2010) and seeks to stake out its own space within linguistic research and highlight its importance within the field of critical discourse studies. It also addresses the discipline's principles, basic areas of inquiry and analytical codes.

Key words: Lexicography, meta-lexicography, ethno-lexicography, critical discourse analysis, ideology, lexicographic discourse, dictionary.

\section{INTRODUCCIÓN}

Este artículo tiene como objetivo presentar y establecer los límites de la lexicografía crítica como reciente disciplina que ha de situarse en el marco general de los estudios etnolingüísticos (Coseriu 1981) por la especial relación que se establece 
entre el diccionario y el modo en que las diferentes culturas organizan y categorizan la realidad y sus distintos dominios de conocimiento. En este sentido, se ha de recordar que ya Dubois (1971: 99) entendía que los diccionarios constituían textes culturels, con lo que el abordaje etnográfico de los repertorios lexicográficos cuenta ya con un largo recorrido. Asimismo, además del amplio marco que proporciona la etnolingüística, es necesario situar esta disciplina en el contexto de los estudios lexicográficos, concretamente metalexicográficos, pues se abordan en ella con mayor profusión los aspectos teóricos relativos al estudio ideológico de los diccionarios, aunque exista una evidente dimensión práctica en la aplicación de los resultados obtenidos a partir del análisis discursivo en las modificaciones realizadas sobre la macroestructura, microestructura e infraestructura del diccionario (Rodríguez Barcia 2016).

Es necesario diferenciar previamente la lexicografía critica de la crítica lexicográfica, ya que se trata de dos disciplinas vinculadas, pero con actividades muy diferentes en cuanto a su metodología y objetivos. Aunque en ambos casos se trata de disciplinas en las que se lleva a cabo la revisión de diccionarios y, en ambos casos también, la perspectiva que adoptan es crítica en cuanto a que se somete una obra a examen crítico, la lexicografía crítica exige por parte de los lexicógrafos y lexicógrafas una forma de ver el mundo comprometida con las ideologías minoritarias y minorizadas con respecto a la cultura dominante (Rodríguez Barcia 2016). Este estudio solo se centrará en la lexicografía crítica.

Investigaciones como la de Pérez (2000: 59) enmarcan los análisis ideológicos y culturales del diccionario en los estudios de etnografía y ya identifican el acercamiento a los repertorios lexicográficos que se lleva a cabo desde la socio-etnolexicografía, bajo la que subyace el principio fundamental de que "los diccionarios, más que puros instrumentos de descripción aséptica y objetiva de las lenguas, son textos que ofrecen una particular e ideológica visión del mundo y de la historia, cultura y sociedad". De hecho, esta monografía de Pérez (2000) se considera pionera en el ámbito hispánico en cuanto al intento de delimitar de forma conjunta el grupo de investigaciones sobre el diccionario en el que este se considera como producto cultural que va más allá de las actividades codificadoras y descodificadoras de la lengua, y el propio autor afirma en el resumen de este trabajo (2000: 150) que

[el libro] se entiende como propuesta inicial para la comprensión cultural de los diccionarios. [...] Diccionarios, discursos etnográficos, universos léxicos quiere insistir en las capacidades del diccionario para referir visiones de la realidad y para definirse como discursos etnográficos que exigen una lectura detrás de la lectura. 
Con todo, la clasificación de los estudios teóricos sobre el diccionario con el foco puesto en los aspectos culturales e ideológicos que propone Pérez (2000) no da cuenta explícitamente de la perspectiva crítica que se asume en este artículo, y se limita a "afinar algunos de los planteamientos teóricos que perfilan esta lectura de los diccionarios como discursos etnográficos y como fuentes para el estudio de las mentalidades y proponer el análisis de algunos casos" (Pérez 2000: 39). El autor refina más su planteamiento teórico en el trabajo de 2005, en el que ya propone los principios que sustentan los estudios etnográficos del diccionario (2005: 9-21), así como sus vínculos con otras disciplinas (2005: 53-64).

Se trata, pues, de profundizar en el estudio etnográfico del diccionario y de proponer un nivel más de concreción a partir de la delimitación teórica de la lexicografía crítica cuya perspectiva analítica no solo tiene en cuenta la realidad cultural e ideológica plasmada en los diccionarios, sino que supera la ya compartida visión del diccionario como producción cultural y se caracteriza como una disciplina sensible y comprometida (al menos en cuanto al ejercicio de denuncia) con los grupos minoritarios y minorizados tradicionalmente por la visión etnocentrista de los repertorios, que suelen trasladar ideologías dominantes. En primer lugar, se enuncian y explican los falsos tópicos generalmente asumidos por la sociedad en cuanto a los diccionarios y a la práctica lexicográfica, puesto que esta disciplina problematiza estas cuestiones generalmente aceptadas por parte de hablantes sin formación especializada en el ámbito filológico y lingüístico. Justamente, como contraposición frente a estos tópicos surgen los principios básicos que sustentan la disciplina. Asimismo, se realiza un resumen del panorama de las investigaciones lingüísticas en las que se tiene como objeto central del estudio el diccionario y su relación con la cultura e ideología. Finalmente, se lleva a cabo en este artículo una exposición breve de la relación que se establece entre diccionario e ideología, así como de la perspectiva y bases metodológicas del análisis crítico del discurso lexicográfico.

\section{DEFINICIÓN Y PRINCIPIOS DE LA LEXICOGRAFÍA}

\section{CRÍTICA}

La lexicografía crítica es la disciplina lingüística, enmarcada en los estudios etnolexicográficos, que se ocupa del estudio y análisis de la ideología en los diccionarios desde una perspectiva comprometida con las minorías y los grupos minorizados, por lo que su crítica se refiere especialmente a las ideologías dominantes. 
Los ejes temáticos principales sobre los que pivota la crítica suelen ser la religión, la política, el sexo, la raza, la clase social y las cuestiones de género.

Como ya advierten Lara (1997: 262) y Svensén (2009: 1), el diccionario es un producto social que juega un papel decisivo en el desarrollo de la cultura, por su carácter de memoria social y como partícipe en la construcción de la sociedad. Si a esto se añade la condición que ha adquirido popularmente de libro total e infalible, depositario de la verdad sobre el mundo, se comprende mejor su relevancia como objeto de estudio desde una perspectiva crítica con las ideologías dominantes y sensible a la representación de los grupos minorizados. Pérez (2005: 13) ya identifica el "carácter sacralizador de la lengua que posee el diccionario" que motiva "la creencia generalizada [de que] los vocablos no registrados por el diccionario terminan no existiendo en la lengua, aunque los hablantes los empleen a diario". Además, puesto que, como ya había identificado Hausmann (1977), las principales funciones del diccionario son dar respuesta a las consultas sobre el léxico de una lengua y servir de herramientas para el aprendizaje lingüístico, se abre aquí la problemática de su carácter referencial y liminar, pues se conforma como puerta de acceso a una lengua, con todo lo que esto conlleva en los procesos iniciales de conocimiento del léxico.

Resumiendo, la condición de herramienta funcional y esencial para el aprendizaje lingüístico (Tarp 2008), su papel añadido como sostén normativo y/o descriptivo de la lengua y el carácter tradicionalmente atribuido de obra poseedora de la verdad ponen a los diccionarios en el punto de mira de los estudios teóricos de la lexicografía desde un posicionamiento alerta en cuanto a las representaciones de la sociedad y, en general, de la realidad, que estos repertorios pueden trasladar. Es en este punto cuando se hace imprescindible una lexicografía crítica.

Como se deduce de la propia definición de la disciplina, en los estudios de lexicografía crítica se incluyen todas aquellas investigaciones en las que se analiza la relación entre ideología y diccionario desde una perspectiva comprometida socialmente con las minorías o grupos minorizados desde la cultura dominante. Los estudios que abordan el análisis conjunto de diccionario, cultura e ideología suelen agruparse bajo la etiqueta general de etnolexicografía (Fillmore 1994; Calero Vaquera 2004). Bajo esta denominación no se encuentran solamente los estudios de lexicografía crítica, sino otros trabajos que se llevan a cabo sin una sensibilidad especial hacia las ideologías no dominantes, pero que se detienen en la presencia de aspectos culturales en los repertorios lexicográficos. Estos constituyen contribuciones a este ámbito de estudio, aunque propiamente se han de enmarcar bajo otras etiquetas como "estudios de diccionario y sociedad", "cultura y diccionario", "diccionario e ideología" o de "diccionario y pragmática", teniendo en cuenta lo que se aborde en cada caso concreto. San Vicente, Garriga y Lombardini (2011: 14) hablan de la incorporación de 
los análisis de la ideología a la crítica lexicográfica, lo que permite entender algunas aportaciones sobre la ideología en el diccionario como un nuevo ítem en la crítica lexicográfica que no tiene que entenderse, necesariamente, como lexicografía critica. Con todo, estos autores son de los primeros en someter a estudio la relación entre diccionario e ideología con carácter monográfico y riguroso en su trabajo plural de 2011, aunque propiamente no identifiquen la noción de lexicografía crítica.

La necesidad de una lexicografía crítica surge como reacción ante algunos tópicos popularmente aceptados de los que se extraen por contraposición los principios que la sustentan. Algunos de estos principios ya fueron esbozados por Pérez (2005), aunque presentados a modo de explicación de las relaciones entre "diccionario e imperfección", "diccionario y sacralidad", "diccionario y verdad" y "diccionario e intencionalidad”. En las líneas que siguen se enuncian, por contraposición frente a los tópicos popularmente admitidos relativos a los diccionarios (especialmente monolingües), los principios fundamentales de la lexicografía crítica y se desmontan las contradicciones inherentes a ellos. Algunos de estos tópicos ya fueron recogidos en Rodríguez Barcia (2016):

\section{PRINCIPIO 1. El diccionario es una obra subjetiva}

Frente al tópico de que el diccionario es una obra descriptiva y neutra, es preciso comprender que se trata de un trabajo sumamente subjetivo. Pérez (2005: 53) señala que "el diccionario no es un libro ingenuo, sino, muy por el contrario, uno en donde la cosmovisión del mundo que señala está, irremisiblemente, condicionada por la mirada subjetiva. Como afirma Forgas Berdet (1996: 78) "toda decisión es ideológica”. Lo cierto es que toda actividad intelectual es ideológica, porque es humana. Toda decisión implica, por tanto, una toma de postura frente al mundo, una visión de la realidad. El diccionario no queda fuera de esta regla y, como producto de la cultura desarrollado por personas con sus especiales idiosincrasias e ideologías, no escapa al hecho de que es una obra que toma partido y es, por ese motivo, parcial. El hecho de que popularmente se conozca al diccionario como una obra descriptiva y neutra convierten su análisis crítico en especialmente relevante, por el valor simbólico que esta obra tiene en casi todas las sociedades y por su consideración de obra objetiva por naturaleza. Frente a la idea de que en las sociedades económicamente desarrolladas existe plena consciencia de los ejercicios de manipulación llevados a cabo a través del discurso, los diccionarios permanecen al margen de esta teoría general, pues la ideología que subyace en estas obras no es intuida por los hablantes sin formación específica. 


\section{PRINCIPIO 2. El diccionario es un producto lexicográfico sujeto a discusión}

Se enuncia frente al tópico de que el diccionario es una autoridad indiscutible, que ya fue sometido a un cuestionamiento profundo en trabajos como el de López Facal (2010). El diccionario, como producto subordinado al uso, no puede constituir per se una garantía lingüística independiente de las prácticas de una comunidad concreta. Esto es, por más que un diccionario se empeñe en orientar preferencias relativas al uso estándar o en desterrar algunos elementos léxicos de una lengua, es el uso el que marca el futuro y, por ello, ha de ser la última voz a la que se debe escuchar cuando se busca la acreditación sobre el léxico de una lengua. A lo largo de las distintas etapas educativas se ha de proporcionar una idea crítica y realista de la esencia naturalmente humana de los diccionarios y permitir que se contradigan y discutan algunas de sus definiciones. Además, por su propia condición de "imperfección" (Pérez 2005: 11), el diccionario se construye como obra discutible, en absoluto inconclusa, es decir, con aspectos contradictorios y sujeta a debate.

\section{PRINCIPIO 3. El diccionario tiene la capacidad de reconstruir la realidad y de promover una determinada cosmovisión}

Al contrario de lo que se suele afirmar en cuanto a que es la realidad la que moldea el diccionario (según palabras de Gutiérrez Ordóñez recogidas por Fanjul 2014), los repertorios lexicográficos también pueden promover y consolidar una determinada visión de la realidad. Si solo fuese la realidad la que moldea el diccionario, todos los diccionarios de una misma lengua serían, en las diferentes épocas, exactamente iguales en cuanto a la ideología proyectada. Es un hecho que esto no es así, como se observa claramente en el caso del español (Rodríguez Barcia 2008). Para una misma realidad, cada diccionario proporciona una definición diferente. Una misma realidad se aborda de maneras distintas según el diccionario, por lo que no es la realidad la que hace al diccionario, sino el diccionario el que la recoge y proyecta del modo que considera más conveniente según la cultura e idiosincrasia de las personas que forman parte del equipo de redacción de las obras. El diccionario sí es y debe ser el reflejo de una cultura, pero ese reflejo puede materializarse de una forma más plural y matizada o más impresionista y particularista, en función del compromiso que las personas redactoras mantengan con la sociedad. Como se viene detectando no solo en español, sino en otras lenguas como el inglés, el diccionario debe superar el etnocentrismo cultural (Moon 2014) y optar por visiones más plurales acordes con una realidad cultural más diversa. 


\section{PRINCIPIO 4. El diccionario es un vehiculo identitario, un aval lingüistico parajuridico y una forma de mediación social}

Este principio se contrapone a la idea reduccionista de que la función principal del diccionario es la de servir de apoyo en el aprendizaje/conocimiento de una lengua. El diccionario es un recurso didáctico que permite conocer mejor una lengua, pero esta no es su función principal. En general, pueden identificarse diversas funciones de los diccionarios fuera de sus evidentes aplicaciones para la corrección lingüística. En el caso del español, la política panhispánica deja claro el poder del diccionario académico como mecanismo de cohesión entre España y el resto de países hispanohablantes. En las lenguas minoritarias, el diccionario posee también ese valor simbólico como pieza que aporta solidez al estándar lingüístico y sobre la que se sustenta, junto con la gramática y la ortografía, la condición plena de lengua. Una función primordial del diccionario monolingüe es, pues, la de servir de obra referencial para la estandarización y cohesión de una lengua. En realidad, más que para favorecer la comprensión, el diccionario monolingüe fue concebido primigeniamente como salvaguarda de un valor esencial de la cultura, la lengua (Lara 1997). Otra función esencial del diccionario monolingüe, ajena al aprendizaje y conocimiento de una lengua, es la de servir como instrumento de decisión con carácter parajurídico en procesos legales de toda índole, desde la redacción de una ley a la sentencia en un tribunal. Enlaza esto con el falso tópico del diccionario como obra incontestable de fiabilidad máxima, un tópico que ignora el carácter humano y, por ello, falible y subjetivo, de una obra que no fue pensada con ese fin, pero que sí es utilizada con esta función de aval. La última de las funciones de los repertorios lexicográficos más allá de su valor para conocer una lengua es la de mediador social. El estatus atribuido popularmente a esta obra permite que se considere una fotografía de la realidad, que cobra una dimensión material a partir de ser nombrada y registrada en el diccionario a través de palabras. Aunque la realidad existe de forma independiente al diccionario, en la consideración tradicional de estas obras se les concede esta función mediadora, y se da así carta de naturaleza a los nuevos usos de una lengua a partir de su inclusión o no en un diccionario, especialmente de una institución de reconocido prestigio, rectora en materia de norma como es la Real Academia Española.

\section{PRINCIPIO 5. Todos los diccionarios, antiguos y contemporáneos, son obras ideologizadas}

No solo los diccionarios antiguos pueden considerarse obras ideologizadas, pese al refinamiento técnico actual suavice notablemente las huellas ideológicas. 
Una lectura descontextualizada de los diccionarios podría proporcionar esta visión simplista. La crítica de los diccionarios del pasado, sobre todo de los diccionarios "de autor" (entre los que se encuentran en el ámbito del español algunos de los mencionados por Álvarez de Miranda 1995), debe hacerse teniendo en cuenta el contexto histórico y lingüístico para romper la falsa creencia de que "obra antigua [es] igual a obra ideologizada” (San Vicente, Garriga y Lombardini 2011: 11). Como los diccionarios actuales, los repertorios realizados en los siglos pasados guardan dependencia con respecto a la ideología de las personas responsables de su redacción. Esto quiere decir que, en la mayoría de casos, es posible detectar huellas de la ideología vertida por estos autores en sus diccionarios. Si a este hecho se une que la técnica lexicográfica estaba menos depurada, sí es posible afirmar que incluso un análisis historiográfico contextualizado de los diccionarios desde el inicio de la lexicografía revela la presencia de ideología en estas obras. Esto no significa que los diccionarios actuales presenten menor carga ideológica. Al contrario, el perfeccionamiento de la técnica lexicográfica ha permitido desarrollar plenamente la labor de la lexicografía práctica, y la presencia de ideología puede entenderse en la actualidad con un mayor nivel de consciencia por parte de los equipos de redacción lexicográfica, tanto si pensamos en diccionarios monolingües como en los bilingües, especializados o incluso en imágenes, con lo que la crítica ha de endurecerse en este sentido.

\section{PRINCIPIO 6. El diccionario puede contribuir al cambio social}

De todos los tópicos anteriores surge la idea de que el diccionario no tiene la capacidad de cambiar la sociedad, aunque esto puede desmontarse fácilmente. La polémica acontecida en 2014 a partir del mantenimiento en el $D R A E$ de la acepción no marcada "trapacero" en la definición de gitano ocasionó en España un sonado movimiento de repulsa por parte de asociaciones como la Fundación Secretariado Gitano, que solicitaba cambios en el diccionario para favorecer el cambio social en cuanto a la inclusión de la comunidad gitana. El diccionario movió a la sociedad a manifestarse, como en otras ocasiones, por lo que generó un cambio social vinculado al respecto de una etnia tradicionalmente rechazada en España. La RAE se comprometió a revisar la definición, y el 13 de octubre de 2015, día en que se puso a disposición pública la Edición del Tricentenario, pudo comprobarse que efectivamente se había modificado, al menos en cuanto a la marcación pragmática de la acepción. Es decir, por coherencia con el uso todavía vigente del sentido despectivo, la acepción se mantuvo, pero con el comentario añadido "U. como ofensivo o discriminatorio”. En otra temática que también atañe a comunidades en riesgo de exclusión, los colectivos de LGTB (lesbianas, gais, transexuales y bisexuales) celebraron 
la modificación llevada a cabo en el diccionario académico al añadir una acepción que reflejaba los cambios en la legislación del matrimonio homosexual. Sin embargo, no tenían en cuenta que la primera acepción de esta voz, la más general, seguía manteniendo la secuencia "unión de hombre y mujer". Que el diccionario mantenga esta primera acepción supone la priorización del matrimonio heterosexual sobre el homosexual. ¿Cambiaría la sociedad por el hecho de cambiar "unión de hombre y mujer" por "unión de personas"? Desde luego que la respuesta es no si se espera un cambio inmediato, pero la respuesta es sí si se tiene en cuenta que una de las fuentes del conocimiento en las primeras etapas formativas es el diccionario y si se analiza como un cambio lento y progresivo. Un simple cambio puede reorganizar la forma de conocer el mundo y de relacionarse con la realidad. Un diccionario puede cambiar el mundo sin necesidad de desvirtuar o falsear la realidad, simplemente ofreciendo una visión respetuosa y diversa al emplear los recursos técnicos que tienen los lexicógrafos y lexicógrafas a su disposición. Asimismo, un diccionario también puede cambiar el mundo en cuanto a generar una reacción en la sociedad, por ejemplo, la que se ha comentado de repulsa ante definiciones discriminatorias.

\section{PRINCIPIO 7. La intervención lexicográfica es posible e, incluso, licita}

Los equipos lexicográficos pueden, y deben, intervenir el diccionario si con ello atiende a demandas sociales emancipadoras. En definitiva, y puesto que se ha admitido que los diccionarios sí pueden promover el cambio social, los lexicógrafos y lexicógrafas pueden modificar el diccionario sin desvirtuar la realidad. Su figura ha de ser proactiva y no pasiva, no solo tendrán un registro notarial de la realidad, sino que podrán favorecer el cambio a partir de las intervenciones precisas en la macroestructura, microestructura e infraestructura de los repertorios. Por ello, la lexicografía crítica se erige como disciplina a caballo entre lo teórico y lo práctico, pues las modificaciones realizadas tendrán que ser implementadas desde la lexicografía práctica.

\section{DICCIONARIO E IDEOLOGÍA: APROXIMACIÓN CONCEPTUAL Y PRINCIPALES INVESTIGACIONES}

Abordar la relación entre diccionario e ideología implica una complejidad teórica por la dificultad que supone interpretar adecuadamente los anclajes de la subjetividad. Uno de los principales problemas de la interpretación ideológica del discur- 
so lexicográfico es precisamente que la mirada crítica es también ideológica; otro, es la falta de aplicación de una metodología unificada y rigurosa. Los instrumentos para el análisis de la ideología han de ser, como advierten San Vicente, Garriga y Lombardini (2011: 13) “necesariamente afinados”, puesto que en el presente se está dando lugar un proceso de transformación ideológica y social que ha de disponer en los contextos adecuados cada obra lexicográfica sometida a análisis. En cualquier caso, lo que no se pone en duda en la actualidad es que el diccionario es una obra transmisora de valores generalmente privativos de determinados grupos sociales y conlleva la proyección de una determinada visión del mundo.

El concepto de ideología se utiliza a veces de forma banal e imprecisa como sinónimo de idiosincrasia, de creencias o, incluso, de cultura. El concepto se ha incorporado desde la literatura especializada hasta el habla cotidiana, pero, como resume Gutiérrez Cuadrado (2011:27), este concepto ha sufrido una evolución importante en su transcurso histórico desde su origen como ciencia de las ideas a partir de Desttut de Tracy — al que se atribuye generalmente el origen moderno del conceptoy su escuela en los primeros años del siglo XIX, hasta su adopción en la doctrina social del materialismo económico de Marx y Engels. Desde el Análisis Crítico del Discurso (en adelante ACD), especialmente en lo que respecta a autores como van Dijk, se considera que la ideología se asienta sobre la psicología y la entienden desde un punto de vista sociocognitivo. Este autor define la ideología como las "creencias fundamentales de un grupo y de sus miembros" (2003: 14). No se trataría de cualquier tipo de creencias socialmente compartidas, sino las axiomáticas, es decir, las que controlan y organizan otras creencias. La comunidad de conocimiento a la que se pertenezca determina la ideología que se traslada al discurso; por ese motivo, no es la misma la ideología que se traslada en las diferentes tradiciones discursivas lexicográficas. En última instancia, desde la perspectiva sociocognitiva de van Dijk (2003) las ideologías son la base de los discursos, por lo que las ideologías serían la base también de los discursos lexicográficos desde esta perspectiva teórica. Según esta orientación la lengua sería el vehículo a partir del que se pondría de manifiesto la ideología, pero es posible poner esto en cuestión y analizar cada signo lingüístico como signo ideológico, sin posibilidad de disociar ambas realidades. De hecho, para superar las debilidades intrínsecas al análisis de la ideología desde la perspectiva sociocognitiva, es necesario negar uno de sus principios, como es el discurso y su capacidad de "control mental", puesto que, en general, en muchos casos no existe desconocimiento por parte de los hablantes de una lengua de la utilización de estrategias lingüística que buscan manipular su comportamiento, sus prácticas. Es más, en el caso del diccionario ya es bien conocida la idiosincrasia de determinadas instituciones y, con todo, es posible realizar un análisis ideológico de los discursos 
lexicográficos en función de la propia naturaleza ideológica del lenguaje. Además, se ha de superar la condición más bien positivista e idealista de este tipo de análisis crítico con perspectiva sociocognitiva, para no solo mostrar el compromiso con las minorías y grupos sociales minorizados, sino con el afán real de intervenir en la lengua y en la elaboración del diccionario si con ello es posible solucionar el tratamiento inicuo de la realidad social desde el lenguaje. Lo cierto es que tanto la mirada ética como la lógica igualitaria que predica el ACD es discutible en cuanto que el individuo es esencialmente un ser ideológico, y por tanto toda referencia a la ética es plenamente subjetiva.

La lexicografía crítica no tiene, ni puede tener, como fin la formulación de contradiscursos ideológicos; es en sí misma una práctica científica de resistencia que ha de superar la mera descripción o denuncia e implicarse con la lexicografía práctica.

En realidad, no ha de entenderse separadamente el lenguaje de la ideología, sino que ambas realidades deben considerarse inseparables; todo signo es ideológico. Antes que el diccionario, a la palabra en general se le ha atribuido un especial potencial en la enunciación de la subjetividad en el lenguaje (Kerbrat-Orecchioni 1986). Esta autora defiende que "toda unidad léxica es, en cierto sentido, subjetiva, dado que las 'palabras' de la lengua no son jamás otra cosa que símbolos sustitutivos e interpretativos de las cosas" (1986: 91). En este mismo sentido, Forgas Berdet habla de ideologemas (1996: 73).

Como consecuencia de la adopción de estas ideas, debe considerarse el diccionario como una entidad ideológica material, ya que no hay nada en él que no sea puramente ideológico a partir de su esencia lingüística. Por lo tanto, no es que la ideología esté presente en el diccionario, sino que forma parte de él, ya que el lenguaje es en sí mismo un producto de la ideología. Teniendo en cuenta este aspecto, tratar de eliminarla o neutralizarla en un diccionario conduce simplemente a su sustitución por otras ideologías que, aun entrando en conflicto con las ideologías dominantes, se convierten progresivamente en las nuevas ideologías dominantes.

\subsection{Las investigaciones de diccionario e ideología}

El estudio conjunto de diccionario e ideología constituye desde los años setenta del siglo XX una tendencia muy prolífica en lo que concierne a la investigación lexicográfica (Beaujot y Lehmann 1978; L’Hermitte 1982; Pascual y Olaguíbel 1992; Alvar Ezquerra 1995; Fishman 1995; Wierzbicka 1995; Forgas Berdet 1996; Rodríguez Barcia 2008; San Vicente, Garriga y Lombardini 2011). En ellos se repite la condición del diccionario como producto cultural e ideológico, así como el hecho de que la presencia de ideología resta veracidad y objetividad a los diccionarios. Con 
todo, a la vista de la indisolubilidad de lenguaje e ideología, la objetividad es más que nunca una utopía. De hecho, el tratar de la subjetividad en el discurso lexicográfico resulta una paradoja, pues el análisis de esa referida subjetividad es por naturaleza, como ya se comentó, igualmente subjetivo. Esto no convierte al análisis crítico en una actividad inútil, sino que en él se reflejaría el enfrentamiento de distintas ideologías, con lo que se habilitaría un motor de desarrollo social.

Algunas investigaciones han entendido el diccionario como una posible arma ideológica con fines proselitistas al servicio de la ideología dominante. Beaujot (1989) da por hecho que en los diccionarios de uso se manifiesta de forma evidente dicha ideología dominante. En el análisis crítico del diccionario existirá siempre una tensión entre la ideología del discurso lexicográfico y la ideología de las personas analistas. Generalmente esta pugna enfrenta ideologías dominantes con ideologías no dominantes, defendidas por las personas que llevan a cabo la lectura crítica del diccionario. Aunque parece obvio que el diccionario no se debe convertir, consciente o inconscientemente, en un producto ideológico al servicio de una determinada ideología o en un arma ideológica, lo cierto es que este género discursivo se ha convertido en un campo de batalla, sobre todo en lo que se refiere a los diccionarios realizados por instituciones de reconocido prestigio o con poder prescriptivo.

Los diccionarios son producto de la cultura (Fillmore 1994), pero también son instrumentos que contribuyen a construir esa cultura (Fishman 1995) y a consolidar prejuicios, tradiciones, estereotipos y, en general, ideas sobre la realidad. Por lo tanto, la condición dual del diccionario como producto y productor de las racionalizaciones de una cultura determinada convierte a los repertorios lexicográficos en un interesante objeto de estudio desde la metalexicografía.

En la actualidad, y como ya se resume en Rodríguez Barcia (2016), es posible distinguir cinco grupos de trabajos que tienen como objeto de estudio la relación que existe entre ideología y diccionario, no todos ellos con perspectiva crítica. Se excluyen de esta relación las aportaciones en las que la perspectiva crítica remite al análisis desde la didáctica de segundas lenguas, como por ejemplo el trabajo de Peters, Tent y Fernández (2006). Asimismo, tampoco se tienen en cuenta los estudios de critica lexicográfica, que se detienen en una suerte de reseña de los repertorios, pero que no suponen investigación en cuanto al análisis crítico de los diccionarios en el sentido que venimos tratando.

En un primer grupo se incluyen trabajos pseudocientíficos, más bien divulgativos, en los que se aborda el análisis de un corpus arbitrario y poco representativo de voces. Generalmente tiene la intención de llamar la atención sobre fenómenos puntuales, definiciones que se han modificado en diferentes ediciones de un mismo diccionario, reclamaciones sociales sobre definiciones que incomodan a determi- 
nados colectivos, etc. Podrían incluirse en este grupo los artículos publicados en periódicos.

En cuanto a las investigaciones científicas, el primer grupo lo conforman las de carácter teórico-metodológico. Estas propuestas buscan sobre todo definir el aparato teórico, conceptual y metodológico que ha de servir de armazón para el estudio ideológico sistemático del diccionario, independientemente de que algunos de ellos muestren sus bases teóricas junto con algunos ejemplos aclaratorios (Pascual y Olaguíbel 1992, Forgas Berdet 1996, Calero Fernández 1999; Pérez 2000; Gutiérrez Cuadrado 2011, Rodríguez Barcia 2012, Camacho Barreiro 2015). En estos trabajos también se recurre a ejemplos reales extraídos de diccionarios, pero sirven generalmente para ilustrar la metodología expuesta. Entre estas hemos de destacar las dirigidas a exponer las bases teóricas de la lexicografía crítica y el encuadre en el paradigma de la lingüística crítica, como es el caso de la propuesta presentada por Chen y Zhao (2014).

Otro grupo de investigaciones está compuesto por trabajos que presentan un análisis dirigido a la descripción de un corpus elegido arbitrariamente, aunque con aspiraciones de representatividad, un enfoque generalmente pragmático y una metodología que, aunque puede ser propia y heterogénea, se aplica de forma sistemática y coherente (Fishman 1995, Wierzbicka 1995, Hornscheidt 2008, Forgas Berdet 2011, Rodríguez Barcia 2008, Moon 2014). En estos estudios se incluyen tanto los que realizan el análisis desde el ACDL como los que no se posicionan con respecto a las ideologías dominantes. Algunos de estos trabajos, como el de Hornscheidt (2008) ya incluyen la etiqueta de "critical Lexicographie".

Algunos estudios, además de la investigación etnolexicográfica propiamente dicha, presentan un glosario comentado y temático de términos recogidos en los diccionarios que entrañan cierta polémica ideológica, sobre todo por centrarse en grupos minoritarios o minorizados. En este último conjunto que hemos definido tendrían cabida trabajos referidos, por ejemplo, al léxico de la religión, el tratamiento racial o de la homosexualidad (Sánchez García 2009, Buzek 2010, Kottelat 2010 y Calero Fernández 2002). En este mismo grupo se pueden considerar también trabajos como el de McBride y Stahl (2009), en el que se hace un ejercicio práctico de lexicografía crítica a partir de la elaboración de un glosario que sirve como soporte del análisis discursivo crítico de otro documento.

Finalmente, hay trabajos que abordan el tema de la ideología en el diccionario de forma tangencial. Es el caso de los estudios que al hilo del análisis de cuestiones relativas a la historia (Esparza Torres 1999) o a la técnica lexicográfica - como la ordenación de acepciones, la marcación u otros aspectos - entran en el terreno de la implicación ideológica que arrastran algunas decisiones de los redactores y redac- 
toras de los diccionarios. También existen abordajes ideológicos circunstanciales, movidos por la consulta recurrente y el análisis de los diccionarios en otras facetas y dimensiones como, por ejemplo, la didáctica (Hwang 2013). Estos estudios no presentan corpus exhaustivos ni representativos, aunque igualmente constituyen aportaciones valiosas en cuanto que completan el panorama de los estudios sobre la ideología que reflejan los diccionarios. En este caso no se trata de investigaciones etnolexicográficas, sino simplemente metalexicográficas en las que se desliza alguna reflexión acerca de aspectos ideológicos y/o culturales.

\section{EL ABORDAJE DESDE EL ANÁLISIS CRÍTICO DEL DISCURSO LEXICOGRÁFICO}

Desde el punto de vista de su perspectiva y metodología analíticas, la lexicografía crítica se vehicula fundamentalmente a través del análisis del discurso y, en concreto, del Análisis Crítico del Discurso Lexicográfico o ACDL (Rodríguez Barcia 2012). Aun teniendo un desarrollo teórico, es incuestionable que los resultados obtenidos a partir del análisis crítico del discurso lexicográfico pueden ser sintetizados y expresados en forma de recomendaciones y deben ser puestos en práctica en la confección de diccionarios, de lo que deviene la dimensión teórico-práctica de esta actividad analítica.

Los estudios críticos del diccionario han de enmarcarse necesariamente en las investigaciones sobre el diccionario entendido como discurso etnográfico, es decir, "como una producción fuertemente cargada de informaciones históricas, sociológicas, ideológicas y etnográficas no siempre evidentes y que, condicionada por ellas, desemboca en una representación de las actitudes mentales de un conjunto humano en un tiempo específico" (Pérez 2000: 23). Sin embargo, aunque adoptamos este marco general que propone Pérez (2000), sí consideramos más productivo acudir a una perspectiva directamente comprometida desde el punto de vista social con los grupos no dominantes. Al igual que Fishman (1995), Pérez entiende una relación circular entre diccionario y cultura que sirve como marco general para el análisis del discurso lexicográfico con orientación crítica.

No entraremos en esta ocasión en la exposición detallada de la metodología analítica de los diccionarios a partir de su estructura y de los enclaves de modalización discursiva que se recoge de forma prolija en Rodríguez Barcia (2012 y 2016).

Centrándonos ya en la perspectiva del ACDL, cabe señalar que este acercamiento a los diccionarios implica comprender en toda su complejidad la relación 
que existe entre discurso lexicográfico y sociedad, así como participar de una perspectiva socialmente comprometida con las ideologías no dominantes en materia religiosa, política y social, con especial interés en la construcción de los discursos dominantes y minimizadores (Wodak 1989; van Dijk 1999). Al igual que a los estudios críticos del discurso, al ACDL le interesan también las estructuras globales de poder (Fairclough 2003), la dimensión histórica del discurso, las cuestiones de género y racismo (van Dijk 1997), la construcción discursiva de la identidad nacional o la relación entre discurso y discriminación.

Las personas que actúan como analistas del discurso suelen proponer soluciones para tratar de neutralizar los aspectos ideológicos presentes en el diccionario, a partir de una actitud crítica o de denuncia social. Esto suele desembocar bien en propuestas destinadas a la reelaboración de determinadas definiciones o al desarrollo de una visión holística del diccionario privada de agentes ideológicos discriminadores, invisibilizadores o minimizadores. Las perspectivas más recurrentes en este punto suelen proponer una visión revisionista o crítica que permite revelar la ideología más o menos implícita en los diccionarios para proceder a reflexionar acerca de sus causas y acerca de las estrategias lingüístico-discursivas que garantizan su integración en el texto lexicográfico. Asimismo, en relación con los contenidos ideológicos, los asuntos tratados suelen tener que ver con aspectos religiosos, políticos o de identidad (género, etnia, clase, etc.). Lo cierto es que esta visión plural e inclusiva también es en sí misma ideológica, y de ahí devienen algunas de las críticas más fundamentadas al ACDL que se comentan en la discusión. Por ello, la consideración más adecuada para proceder al análisis crítico del discurso lexicográfico es la que no entiende separación entre lenguaje e ideología, sino la consideración de la condición inseparable de ambas realidades, ya que todo signo es ideológico y, al mismo tiempo, todo lo ideológico va a tener un valor semiótico. Con todo, aunque a partir de esta reflexión parezca poco interesante o productivo realizar un análisis crítico del diccionario, o de cualquier otro producto de la cultura, lo cierto es que sigue siendo imperativo buscar soluciones en cuanto a la orientación ideológica de algunos diccionarios. Esto es, aun erigiéndose en adalides de lo políticamente correcto, con todas las contradicciones que implica asumir, las personas que aborden el análisis crítico de los repertorios lexicográficos deben plantear nuevos caminos que comporten avances ideológicos. En otras palabras, sí tiene sentido realizar un análisis crítico del diccionario, pues en esta obra se proporciona a partir de lo lingüístico-ideológico el medio óptimo para la lucha ideológica. Por todo ello, es esencial que los analistas tomen partido desde el punto de vista ideológico, es decir, que se posicionen, y no se limiten a plantear una ingenua y utópica neutralidad de los diccionarios o un vano intento por neutralizar la ideología. 


\section{DISCUSIÓN Y CONCLUSIONES}

Tradicionalmente, los estudios lingüísticos y, en especial, los sociolingüísticos no han tenido en cuenta el diccionario como objeto de estudio esencial en la sociedad, como elemento que necesita de un análisis más allá de la descripción técnica o de la crítica lexicográfica. En esta disciplina es esencial realizar el análisis a la luz de una perspectiva sensible con la minorización o infrarrepresentación de realidades religiosas, étnicas, sexuales y sociales en función de la cultura mayoritaria que sirve como marco a la elaboración de repertorios lexicográficos. Esto no quiere decir que se parta de una consideración cognitivista que entienda que el diccionario sirve como instrumento para programar a los sujetos e inocularles una determinada ideología, sino que considera el diccionario como una herramienta más de las ideologías dominantes vinculadas a los organismos de poder que organizan y orientan en general la forma de ver y de estar en el mundo.

Moralejo Silva (2017) hace una crítica de la aproximación que los estudios críticos del discurso realizan al análisis de la ideología en los discursos, entre los que también se entendería el discurso lexicográfico. En concreto, considera que las premisas teóricas de sus principales representantes conducen a "callejones sin salida" (2017: 223) y que su motivación crítica permite únicamente plantear "contra-discursos" (2017: 231). Por ello, pese a entender el lenguaje como un fenómeno imposible de desligar de la sociedad, este investigador entiende que la perspectiva principal del ACD es "positivista-idealista" (2017: 235) y que sería preciso explorar otras dinámicas de análisis ideológico, entendiendo de forma diferente la ideología en la línea de pensamiento de autores como Žižek (2010). En general, desde esta postura todo acercamiento crítico al análisis del discurso con base en la noción de ideología de corte cognitivista se enfrentaría no solo a limitaciones metodológicas y teóricas, sino que quedaría subsumida en el vacío de los compromisos voluntaristas y solamente produciría resultados de denuncia social que, en realidad, no ofrecerían información que fuese realmente desconocida por el conjunto de hablantes. Esto es, según esta perspectiva, se da por hecho que el conjunto de la sociedad es consciente de las manipulaciones a las que están sometidos a través del discurso, por lo que la labor analítica crítica sería casi innecesaria. Lo cierto es que este argumentario funciona, en general, para multitud de géneros discursivos, como la comunicación interna y externa de las empresas o el discurso publicitario y político. Sin embargo, en el caso del discurso lexicográfico, la condición casi reverencial que el diccionario ha adquirido en la sociedad y su carácter popular de producción infalible e indiscutible, convierten este género en una excepción a las críticas del abordaje analítico desde el ACD. En otras palabras, en el caso de los diccionarios sí es necesario arrojar 
luz a los hablantes de una lengua sobre el ejercicio de construcción y proyección de una determinada cosmovisión a través de los diccionarios, que pasa desapercibida salvo para las personas especializadas en materia filológica o lingüística. Por ello, la Lexicografía crítica se convierte en el contexto sociopolítico actual en una disciplina clave.

A lo largo de la historia, el diccionario ha sido testigo y cronista de los diferentes procesos de cambio social. Esta dimensión pasiva de la relación entre diccionario y cambio social se enfrenta con una nueva faceta de la lexicografía como impulsora del cambio. La lexicografía ha de ser proactiva, ha de analizar el estado de la sociedad en el momento en el que se compone un diccionario y ha de actuar de forma acorde con las necesidades que sean previsibles a corto y medio plazo. Es importante, pues, llamar la atención sobre la necesidad de intervenir el diccionario para que sea más que un mero notario o cronista, más que un mediador parcial movido por ideologías dominantes, y que se convierta, en definitiva, en un verdadero motor del cambio social.

En conclusión, las investigaciones realizadas hasta el momento en cuanto a la presencia de ideología en los diccionarios han proporcionado materiales muy relevantes para la revisión y actualización de los repertorios lexicográficos. La heterogeneidad de estos estudios no ha impedido que se hayan convertido en impulsores de una nueva forma de entender los diccionarios y su relación con la sociedad y la cultura. Pese a las debilidades que entraña todo análisis ideológico, la lexicografía crítica está llamada a convertirse en una disciplina esencial en los estudios lingüísticos que ya no pueden desligarse en nuestro tiempo de las distintas problemáticas y demandas sociales.

\section{REFERENCIAS BIBLIOGRÁFICAS}

ÁLVAREZ DE MIRANDA, P. (1995): “Hacia una historia de los diccionarios españoles en la edad moderna”. Bulletin Hispanique, 97, 1, 187-200.

BEAJOUT, J. P. y M. LEHMANN (1978): “Idéologie de la langue et idéologie du dictionnaire". Bulletin du centre d'analyse du discours de l'Université de Lille, 3, 163-165.

BUZEK, Ivo (2010): La imagen del gitano en la lexicografía española. Brno: Masarykova Univerzita.

CALERO FERNÁNDEZ, M. á Á. (1999): “Diccionario, pensamiento colectivo e ideología o los peligros de definir”. En N. Vila, M. á Á. Calero, R. Mateu, M. Casanovas y J. L. Orduña (eds.), Asi son los diccionarios. Lleida: Servei de Pu- 
blicacions de la Universitat de Lleida, 149-201.

CALERO FERNÁNDEZ, M. á. (2002): "Homosexualidad y heterosexualidad en los diccionarios: ¿tabú lingüístico o cuestión de género?”. En A. M. ${ }^{a}$ Vigara Tauste y R. M. Jiménez Catalán (eds.), Género, sexo y discurso. Madrid: Ediciones del Laberinto, 47-101.

CALERO VAQUERA, M. ${ }^{a}$ L. (2010): "Ideología y discurso lingüístico: la Etnortografía como subdisciplina de la glotopolítica”. Boletín de Filología, XLV, 2, 31-48

CAMACHO BARREIRO, A. (2015): Marcas ideológicas en una muestra del discurso de las definiciones en la lexicografía cubana de los siglos XIX y XX: identifcación y funcionalidad. La Habana: Editorial Universitaria.

CHEN, W. y Y. ZHAO (2014): "Modern Lexicography: A critical perspective”. Modern Foreign Languages, 2014, 2, s. p. [Disponible en http://en.cnki.com. cn/Article_en/CJFDTotal-XDWY201402006.htm].

COSERIU, E. (1981): “La socio- y la etnolingüística. Sus fundamentos y tareas”. Anuario de Letras, XIX, 5-30.

DUBOIS, J. y C. DUBOIS (1971): Introduction à la lexicographie: le dictionnaire. Paris: Larousse.

ESPARZA TORRES, M. Á. (1999): “Notas sobre el Diccionario Nacional de Ramón Joaquín Domínguez”. En M. C. Henríquez y M. Á. Esparza (eds.), Estudios de Historiografía Lingüistica Hispánica. Vigo: Universidad de Vigo, 39-63.

FAIRCLOUGH, N. (2003): Analysing Discourse: Textual Analysis for Social Research. London: Routledge.

FANJUL, C. (2014): "Es la realidad la que moldea el nuevo diccionario, la RAE no hace ideología”. Diario de León, 26-10-2014, s. p. [Disponible en http:// www.diariodeleon.es/noticias/cultura/es-realidad-moldea-nuevo-diccionario-rae-no-hace-ideologia_930989.html].

FILLMORE, Ch. (1994): "Lexicography and Ethnographic Semantics". En W. Martin, W. Meijs, M. Moerland, E. ten Pas, P. van Sterkengurg y P. Vossen (eds.), Euralex 1994 Proceedings. Ámsterdam, s. n. [Disponible en: http://euralex.org/category/publications/euralex-1994/].

FISHMAN, J. (1995): "Dictionaries as culturally Constructed and as Culture-Constructing Artifacts: The Reciprocity view as seen from Yiddish”. En H. Kahane y B. Kachru, (eds.), Cultures, Ideologies and the Dictionary. Studies in Honor of Ladislav Zgusta. Tubinga: Niemeyer, 29-35.

FORGAS BERDET, E. (1996): “Lengua, sociedad y diccionario: La ideología”. En E. Forgas Berdet (coord.), Léxico y diccionarios. Tarragona: Universitat Rovira 
i Virgili, 71-90.

FORGAS BERDET, E. (2011): "El compromiso académico y su reflejo en el DRAE: los sesgos ideológicos (sexismo, racismo, moralismo) del diccionario". En S. Senz y M. Alberte (eds.), El dardo en la Academia. Barcelona: Melusina, 425-459.

GUTIÉRREZ CUADRADO, J. (2011): “Ideología y lexicografía”. En F. San Vicente, C. Garriga y H. Lombardini (coords.), Ideolex. Estudios de lexicografía e ideología. Monza: Polimetrica, 25-66.

HAUSMANN, F. J. (1977): Einführung in die Benutzung der neufranzösischen Wörterbücher. Tubinga: Niemeyer.

HORNSCHEIDT, A. (2008): "A concrete research agenda for critical lexicographic research within critical discourse studies: an investigation into racism/ colonialism in monolingual Danish, German, and Swedish dictionaries". Critical Discourse Studies, 5, 2, 107-132.

HWANG, A. D. (2013): "Lexicographie française: une approche critique". Synergies Brésil, 11, 105-112.

KOTTELAT, P. (2010): "Définitions lexicographiques et idéologie: ambiguïtés discursives dans les définitions des races, traces de permanence de stéréotypes racistes?”. Publif@rum 10, s. p. [Disponible en http://www.publifarum.farum.it/ezine_pdf.php?art_id=119].

L'HERMITTE, R. (1982): “Lexicographie et idéologie”. Revue des Études Slaves, LIV, 3, 403-408.

LARA, L. F. (1997): Teoría del diccionario monolingüe. México: El Colegio de México.

LÓPEZ FACAL, J. (2010): La presunta autoridad de los diccionarios. Madrid: CSIC/Catarata.

MCBRIDE, N. y B. C. STAHL (2009): “Egypt's Information Society Strategy: A Critical Lexicography". Journal of International Technology and Information Management, 18, 1, 1-33.

MOON, R. (2014): “Meanings, Ideologies, and Learners' Dictionaries”. En A. Abel, C. Vettori y N. Ralli (eds.), Proceedings of the XVI EURALEX International Congress. The User in Focus. Bolzano: EURAC/Institute for Specialised Communication and Multilingualism, 85-105. [Disponible en http://www. euralex.org/elx_proceedings/Euralex2014/euralex_2014_004_p_85.pdf].

MORALEJO SILVA, R. (2017): Ideologia, crisis y desvalorización de la fuerza de trabajo: la industria de la automoción en Vigo. Vigo: Universidade de Vigo. Tesis doctoral inédita. [Disponible en http://www.investigo.biblioteca.uvigo.es/ xmlui/handle/11093/818]. 
PASCUAL, J. A. y M. ${ }^{a}$ C. OLAGUÍBEL (1992): “Ideología y diccionario”. En I. Ahumada Lara (ed.), Diccionarios españoles. Contenido y aplicaciones. Lecciones del I Seminario de Lexicografía Hispánica. Facultad de Humanidades, Jaén, 21 al 24 de enero de 1991. Jaén: El Estudiante, 73-89.

PÉREZ, F. J. (1997): Estudios de lexicografía venezolana: Historia y lexicografía antigua, metalexicografía y etnolexicografía. Caracas: La Casa de Bello.

PÉREZ, F. J. (2000): Diccionarios, discursos etnográficos, universos léxicos: propuestas teóricas para la comprensión cultural de los diccionarios. Caracas: Universidad Católica Andrés Bello.

PÉREZ, F. J. (2005): Pensar y hacer el diccionario. Nociones de lexicografia: definiciones, géneros, critica e historia. Criterios para elaborar diccionarios, métodos de investigación y bibliografía lexicográfica. Caracas: Los libros de El Nacional.

PETERS, P., J. TENT y T. FERNÁNDEZ (2006): “Critical Lexicography”. En E. Corino, C. Marello y C. Onesti (eds.), Atti del XII Congresso Internazionale di Lessicografia. Torino, 6-9 settembre 2006. Proceedings of XII Euralex International Congress. Alessandria: Edizioni dell'Orso, vol. 1, 561-565.

RODRÍGUEZ BARCIA, S. (2008): La realidad relativa. Evolución ideológica en el trabajo lexicográfico de la Real Academia Española (1726-2006). Vigo: Servizo de Publicacións Universidade de Vigo.

RODRÍGUEZ BARCIA, S. (2012): "El análisis ideológico del discurso lexicográfico: una propuesta metodológica aplicada a diccionarios monolingües del español". Verba. Anuario Galego de Giloloxia, 39, 135-159.

RODRÍGUEZ BARCIA, S. (2016): Introducción a la lexicografía. Madrid: Síntesis.

SAN VICENTE, C. GARRIGA y H. LOMBARDINI (2011): “Aproximación metodológica al estudio de la ideología en los diccionarios”. En ídem (coords.), Ideolex. Estudios de lexicografía e ideología. Monza: Polimetrica, 9-24.

SÁNCHEZ GARCÍA, F. J. (2009): El léxico religioso en los diccionarios de la Real Academia Española. Estudio diacrónico. Barcelona: Universitat de Barcelona.

SVENSÉN, B. (2009): A Handbook of Lexicography. The Theory and Practice of Dictionary-Making. Cambridge: Cambridge University Press.

TARP, S. (2008): Lexicography in the Borderland between Knowledge and Non-Knowledge. General lexicographical theory with particular focus on learner's lexicography. Tubinga: Niemeyer.

VAN DIJK, T. A. (1999): "El análisis crítico del discurso". Anthropos, 186, 23-36.

VAN DIJK, T. A. (2003): Ideología y discurso. Barcelona: Ariel Lingüística.

WIERZBICKA, A. (1995): "Dictionaries and ideologies: three examples from Eastern Europe”. En B. B. Kachru y H. Kahane (eds.), Cultures, Ideologies and 
the Dictionary. Studies in Honor of Ladislav Zgusta. Tubinga: Niemeyer, 181-195.

WODAK, R. (ed.) (1989): Language, Power and Ideology: Studies in Political Discourse. Ámsterdam: John Benjamins [Critical Theory, 7].

ŽIŽEK, S. (2010): El sublime objeto de la ideologia. Madrid: Siglo XXI.

Fecha de recepción: 17 de febrero de 2018 Fecha de aceptación: 25 de marzo de 2018 
